\title{
Measurement of Spatiotemporal Structures of Density Fluctuations Using Two-Directional Beam Emission Spectroscopy in LHD
}

\author{
Makoto ONO, Katsumi IDA ${ }^{1)}$, Mikirou YOSHINUMA ${ }^{1)}$, Haruhisa NAKANO ${ }^{1)}$, \\ Tatsuya KOBAYASHI ${ }^{1)}$ and Chanho MOON ${ }^{2)}$ \\ Department of Fusion Science, School of Physical Sciences, SOKENDAI (The Graduate University for Advanced Studies), \\ Toki, Gifu 509-5292, Japan \\ ${ }^{1)}$ National Institute for Fusion Science, Toki, Gifu 509-5292, Japan \\ ${ }^{2)}$ Max-Planck-Institut für Plasmaphysik, EURATOM Association, 85740 Garching, Germany
}

(Received 6 January 2016 / Accepted 26 May 2016)

\begin{abstract}
A beam emission spectroscopy (BES) system for density fluctuation measurements having the sight lines passing through the plasma in the toroidal direction was developed in the Large Helical Device (LHD). The coverage of the area sampled by $15 \times 100$ optical fibers is nearly from the core to the edge on the horizontally elongated poloidal cross section with the spatial pitch of around $1.0 \mathrm{~cm}$, and 32 channels is detected simultaneously with the $4 \times 8$ pixel Avalanche Photodiode Detector camera. In order to improve the directional sensitivity for the propagation of the density fluctuation in the radial direction or the poloidal direction, we propose the use of slit-shaped sight lines. An initial result of the density fluctuation measurements for MHD activity in the edge region and its cross-correlation analysis is presented.
\end{abstract}

(c) 2016 The Japan Society of Plasma Science and Nuclear Fusion Research

Keywords: plasma diagnostic, beam emission spectroscopy, density fluctuation, magnetohydrodynamics, Large Helical Device

DOI: $10.1585 /$ pfr.11.1402115

\section{Introduction}

As a method for a multi channel local measurement of density fluctuations, Beam Emission Spectroscopy (BES) [1] has been widely used in fusion experimental devices $[2,3]$. The BES system consists of Neutral Beam Injection (NBI) for emitting light (Beam emission), optical fibers for taking an image, spectroscopic devices to distinguish the beam emission, and detectors for fluctuating signals. Turbulence occurs along magnetic lines, and thus turbulence has inherently two-dimensional dynamics on the plane vertical to the magnetic field. BES has been applied to investigate into space correlations in radial/poloidal directions and also to visualize the two-dimensional turbulence field in some tokamaks [4,5]. Although it is difficult to apply BES to the non-axis symmetric magnetic fields due to their complicated magnetic fields configuration, BES systems also have been developed in some helical devices [6-8]. The BES system was applied to the Compact Helical Device (CHS) for the first time among helical devices. With an optimized radial spatial resolution by using sight lines nearly tangent to the magnetic axis, the simultaneous measurement of the density fluctuations and the density gradient was accomplished [9]. Because of the larger size of the Large Helical Device (LHD) [10] compared with CHS, the distance from diagnostic ports to the plasma is longer and the solid angle for collecting beam emissions is smaller. Therefore, it is necessary to increase the sampling area to

author'se-mail: ohno.makoto@nifs.ac.jp achieve sufficient detected photon flux and signal-to-noise ratio for fluctuation spectral analysis. In most conventional BES systems, a sampling area is a round shape due to the shape of a fiber, leading to the same sensitivity for waves in radial and poloidal directions. To make a rectangular shape of the sampling area, several fibers are bundled in several BES systems $[3,11]$. The BES system in LHD has been applied to a new fiber bundle design which yields slit-shaped sampling images on the focal plane. This new design has the disadvantage of lower spatial resolution in the direction along the long sides of the slit. However, the design of the fiber bundles has higher wave number sensitivity in the direction along the narrow sides of the slit compared with a round- or square-shaped sampling image. In this system, poloidally-elongated slits are aligned in radial direction for measuring the radially propagating density fluctuations while radially-elongated slits are aligned in poloidal direction for measuring the poloidally propagating density fluctuations. This article presents the system configuration of BES measurement in LHD and initial results of the spatio-temporal structure analysis using magnetic fluctuation as a supporting signal.

\section{BES System in LHD}

In the BES measurement, light emitted from the collisionally excited neutral beam atoms (beam emission) in the intersection of the beam line and the sight line is detected. The fluctuations of the beam emissions are regarded as the 


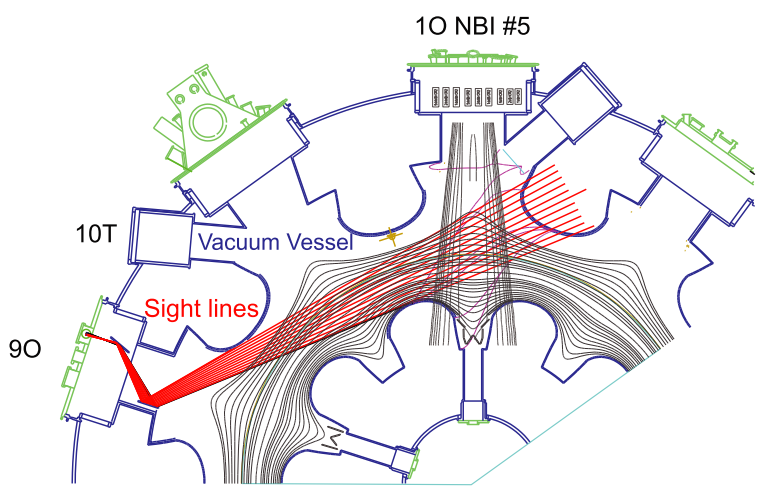

Fig. 1 Top view of the sight lines of the BES system in LHD. Red lines are some of the sight lines on the equatorial plane.

local density fluctuations. In the present experiment, $\mathrm{H}_{\alpha}$ emission from excited neutral beam atoms was measured as beam emission. In LHD, several BES systems have been developed for the local measurement of the density fluctuations. The BES system presented in this paper observes the density fluctuations on the poloidal cross-section. One of the most characteristic aspects is that the sight lines of this system can be optimized to investigate propagation behaviors for radial and poloidal direction by making slitshaped measurement channels perpendicular to each direction. Figure 1 shows the top view of LHD drawn with the BES sight lines, the probe beam (NBI No. 5), and the typical magnetic surface on the equatorial plane. The sight lines pass throughout the plasma in the toroidal direction, aligned for the radial and vertical directions. A neutral hydrogen atomic beam for heating is used as the probe beam. The angles between the sight lines and the beam line range from $113^{\circ}$ to $120^{\circ}$ on the equatorial plane. The beam energy is $40 \mathrm{keV}$ in these experiments, which yield a Doppler shift of $2.3 \sim 3.0 \mathrm{~nm}$ in the $\mathrm{H}_{\alpha}$ beam emission for those angles.

Figure 2 (a) shows the measurement positions where the sight lines intersect the beam line center in the poloidal cross-section. Each fiber has a numerical aperture of 0.25 , a core diameter of $400 \mu \mathrm{m}$, and a clad diameter of $420 \mu \mathrm{m}$. The fiber images are almost focused at the center of the neutral beam and the roughly 25-to-1 magnification results in an observed spot size of about $1 \mathrm{~cm}^{2}$ in a poloidal cross section. The spots are located immediately adjacent to each other in each direction, therefore the spacial pitch $\Delta x \sim 1.0 \mathrm{~cm}$ for radial/vertical direction. The lights collected from the plasma are transmitted through the fibers to two grating spectrometers. The beam emission separated from the background emission is detected by using APDCAM which is a $4 \times 8$ pixel Avalanche Photodiode Detector camera with a sampling rate of $100 \mathrm{kHz}$. The arrangement of the fibers connected to the detection system can be selected, and the beam emission is detected with chosen 32 channels simultaneously. For the experiment discussed

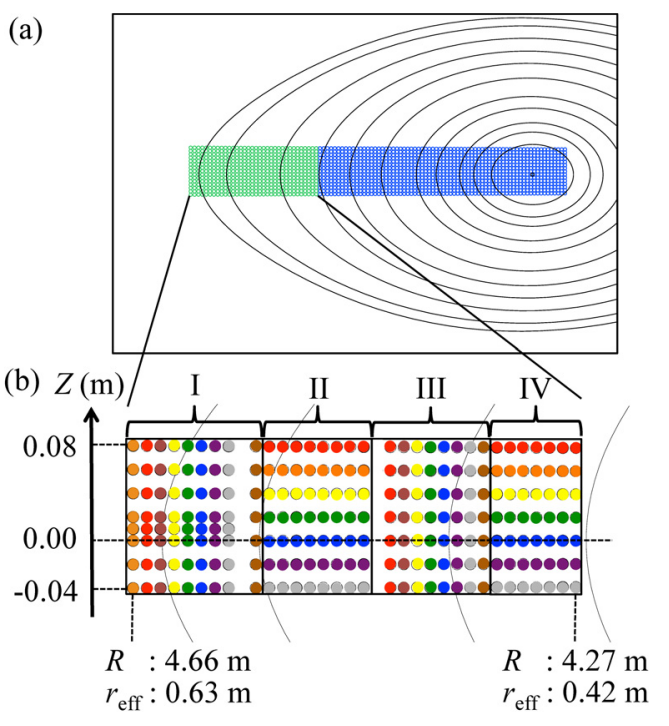

Fig. 2 (a) Focal images of optical fiber array on the poloidal cross section along the probe beam (NBI\#5). (b) Fiber bundle configuration utilized in the experiment. Seven or eight fibers are bundled together to make radially-aligned channel arrays (groups I and III) and poloidally-aligned channel arrays (groups II and IV).

here, the outer region of $0.42<r_{\text {eff }}<0.63 \mathrm{~m}$ is of interest, as shown in Fig. 2 (a) with green spots. Here, $r_{\text {eff }}$ is the averaged minor radius on a magnetic flux surface. To optimize the BES system to investigate into the fluctuation propagation in the poloidal cross-section, some fibers are bundled to make a slit-shaped sight line to improve the signal to noise ratio, as shown in Fig. 2 (b). The fibers which correspond to the same colored spots make each channel. The vertically accumulated slit sight lines (aligned in the radial direction) yield higher sensitivity for a radial propagation, though the spatial resolution is lowered in the vertical direction. The radially accumulated slit sight lines yield higher sensitivity for a poloidal propagation.

\section{Experiment}

\subsection{Low-frequency density fluctuations in the edge region}

Experimental observations are carried out in the LHD, which has a major radius $R$ of $3.5 \mathrm{~m}$ and an averaged radius of $0.6 \mathrm{~m}$. The measurement presented in this paper was obtained in high $\beta$ plasma, where the toroidal magnetic field strength at the magnetic axis $\left(R_{\mathrm{ax}}=3.575 \mathrm{~m}\right)$ $B_{\text {ax }}$ was $1.00 \mathrm{~T}$, and the magnitude of the quadrupole field $B_{\mathrm{q}}$ was $100 \%$. Figure 3 shows the typical experimental conditions and temporal evolution of plasma parameters for a discharge in which the low-frequency fluctuation appears: (a) the NBI powers, (b) the line-averaged density, (c) the electron temperature, (d) the beam emission, and (d) the magnetic fluctuation. Additional gas was puffed to increase density. The target high $\beta$ plasma which is produced 


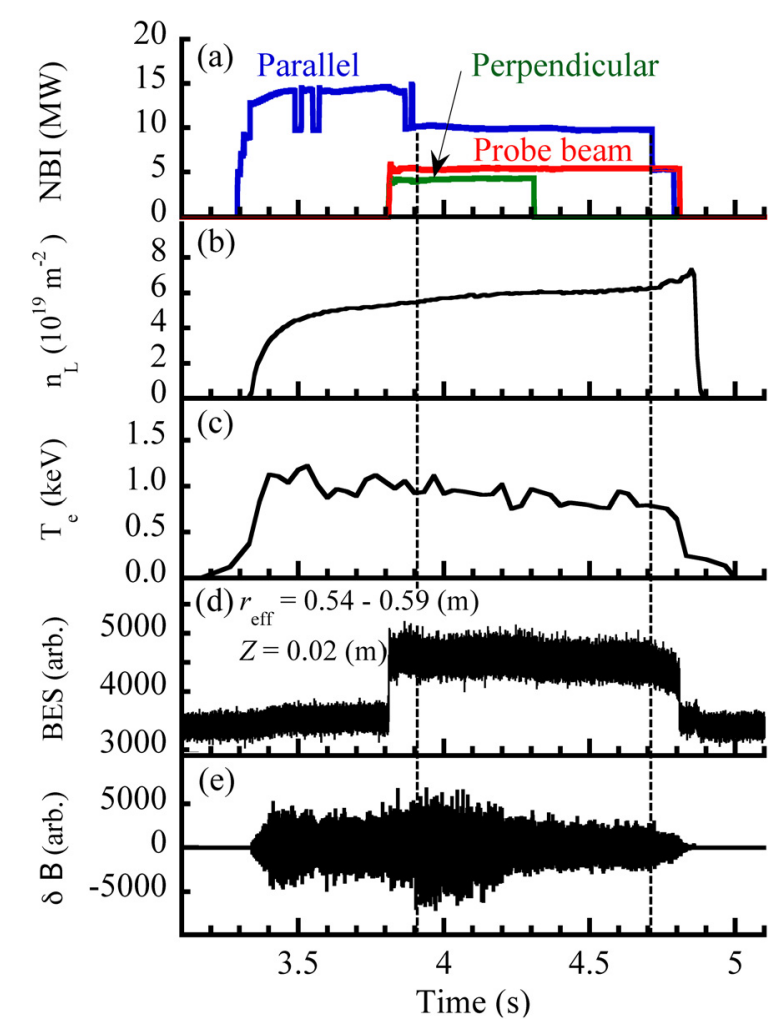

Fig. 3 A typical temporal evolution of plasma parameters for a discharge in which low-frequency fluctuations appear (\#120280): (a) the NBI powers, (b) the line-averaged density, (c) the electron temperature, (d) the beam emission, and (e) the magnetic fluctuation.

with two tangential NBIs of a total power of $10 \mathrm{MW}$, and a perpendicular NBI of 5.4 MW and another perpendicular NBI of 4.3 MW was superimposed for $3.81<t<4.38 \mathrm{~s}$.

Correlation analysis is applied during the quiet period in $3.91<t<4.71 \mathrm{~s}$, which is shown by the vertical lines in Fig. 3. During this period, the line-integrated density $\left(n_{\mathrm{L}}\right)$ increased from $5.4 \times 10^{19}$ to $6.3 \times 10^{19} \mathrm{~m}^{-3}$, while the central electron temperature slightly decreased from 1.0 to $0.9 \mathrm{keV}$.

The density fluctuations are observed with the BES. Figure 4 (a) demonstrates the time averaged auto-power spectrum of the beam emission measured with a channel of group II at $r_{\text {eff }} \sim 0.57$ (blue line) for $3.9<t<4.7 \mathrm{~s}$, together with the spectrum of noise signal (green line). The noise signal was measured in the period when the probe neutral beam was not injected and was the background $H_{\alpha}$ light. The frequency peak of the beam emission was observed at around $2.8 \mathrm{kHz}$, while those of the magnetic fluctuation $\tilde{B}$ were at $2.4 \mathrm{kHz}$ and $4.8 \mathrm{kHz}$, as shown in Fig. 4 (b).

The level of density fluctuations was quite weak. However, the BES signals have unambiguous crosscorrelation with magnetic probe signals. In Fig. 5, the squared cross-coherence shows a peak at $f \sim 2.6 \mathrm{kHz}$. High cross-correlation between the density and magnetic

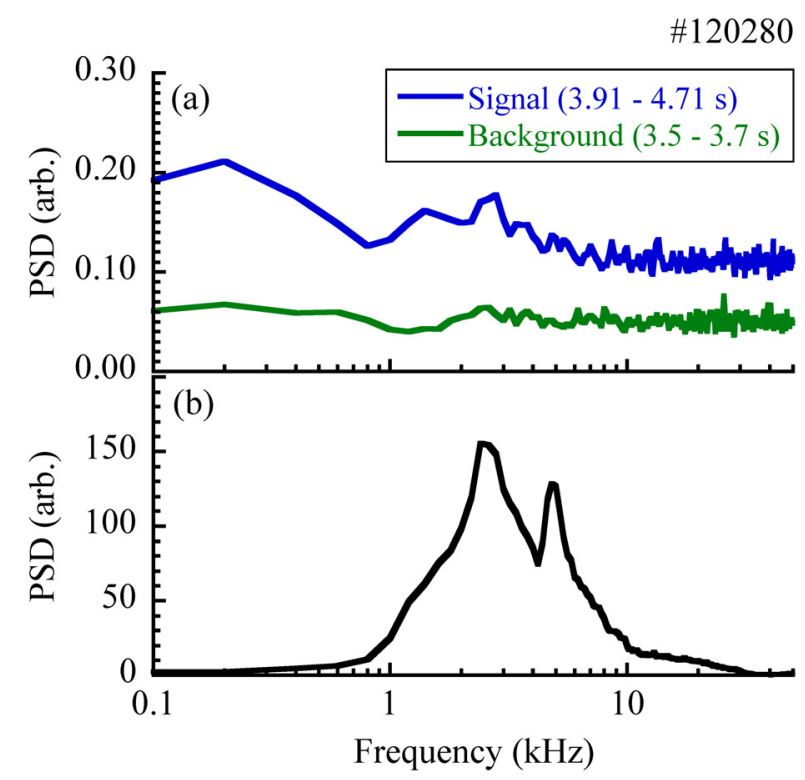

Fig. 4 Fourier power spectrum of (a) the density fluctuation $\left(0.54<r_{\text {eff }}<0.59 \mathrm{~m}, \mathrm{Z}=0.02 \mathrm{~m}\right)$ and $(\mathrm{b})$ the magnetic fluctuations in $3.909-4.709 \mathrm{~s}$.

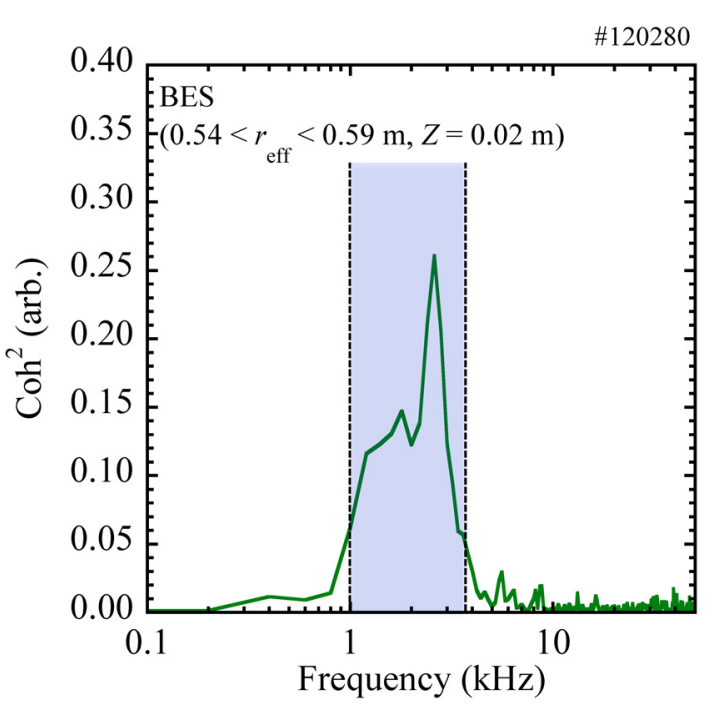

Fig. 5 Squared coherence between the density and magnetic fluctuations in $3.909-4.709 \mathrm{~s}\left(0.54<r_{\mathrm{eff}}<0.59 \mathrm{~m}, Z=\right.$ $0.02 \mathrm{~m}$ ). The radial profile of the coherence in the range of $1.0-3.5 \mathrm{kHz}$ is shown in Fig. 6 .

fluctuations shown here indicates that the oscillation may be MHD modes.

The radial profile of the coherence for the low frequency mode $(1.0-3.5 \mathrm{kHz})$ is shown in Fig. 6. The diamond and circle symbols in this figure indicate the BES channels of the radially-aligned arrays (groups I and III), and the triangle and square symbols indicate the BES channels on $Z=0.02 \mathrm{~m}$ of the poloidally-aligned arrays (groups II and IV). Significant coherence values are observed at $r_{\text {eff }} \sim 0.57 \mathrm{~m}$. 


\subsection{Spatio-temporal structure}

Spetio-temporal structure of the fluctuation has been used to investigate the propagation behavior of the mode. In this analysis, the magnetic signal is used as a reference, and this leads to more distinct results due to its high in-

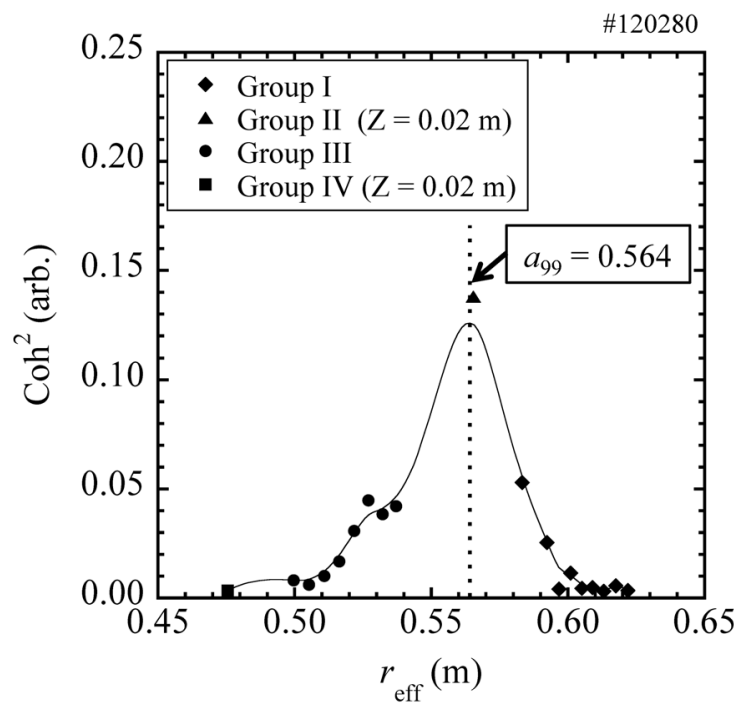

Fig. 6 The radial profile of the squared coherence between the density fluctuation measured and the magnetic fluctuation of the low-frequency component $(1.0-3.5 \mathrm{kHz})$. The value of coherence peaks at near $r_{\mathrm{eff}}=a_{99}=0.564$, where $a_{99}$ means the effective minor radius inside which $99 \%$ of the electron kinetic energy exists. tensity. Spatiotemporal structure of density fluctuations is determined by a two-point two-time correlation function at a different position. Two-point two-time correlation function between BES signals and the magnetic signals can be given by

$$
R(\xi, \tau)=\frac{\left\langle f(\xi, t) f_{\mathrm{ref}}(t+\tau)\right\rangle}{\sqrt{\left\langle f^{2}(\xi, t)\right\rangle} \sqrt{\left\langle f_{\mathrm{ref}}^{2}(t)\right\rangle}},
$$

where $\tau$ is the time lag, $f(\xi, t)$ is the time series of the BES channel located at $\xi, f_{\text {ref }}(t)$ is the time series of the magnetic fluctuation, and \langle\rangle stands for temporal average, defined as $\langle h(t)\rangle=(1 / T) \int_{0}^{T} h(t) \mathrm{d} t$.

Figures 7 (a) and (b) show a contour plot of correlation functions of the density fluctuation (which is band-passed in the frequency range of $1.0-3.5 \mathrm{kHz}$ ) at 7 poloidallyaligned BES channels (group II) and 8 radially-aligned BES channels (group III), respectively, with the magnetic fluctuation as a reference signal. The correlation functions are calculated with a time window of $3.0 \mathrm{~ms}$ and are ensemble-averaged for 265 samples during the quiet period in $3.911<t<4.706 \mathrm{~s}$. In Fig. 7 (a), the vertical direction $Z$ almost corresponds to the poloidal direction at the location of the BES sample volume.

For the fluctuation component at $f \sim 2.6 \mathrm{kHz}$ at $r_{\text {eff }} \sim 0.57 \mathrm{~m}$, the fluctuation structure is found to propagate in the electron diamagnetic drift direction which corresponds to the $E \times B$ flow measured with the charge exchange spectroscopy. In the radial inside of $r_{\mathrm{eff}} \sim 0.57 \mathrm{~m}$, (a)

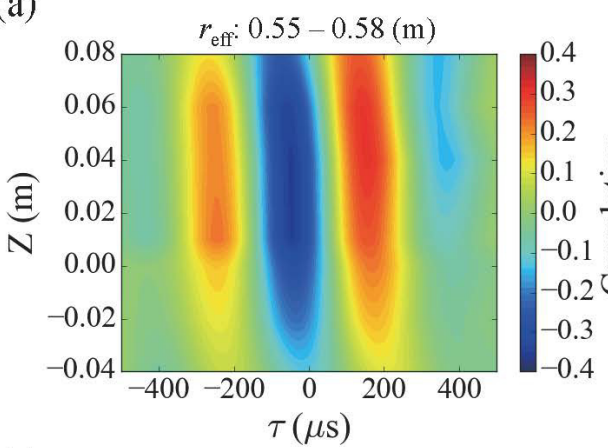

(c)

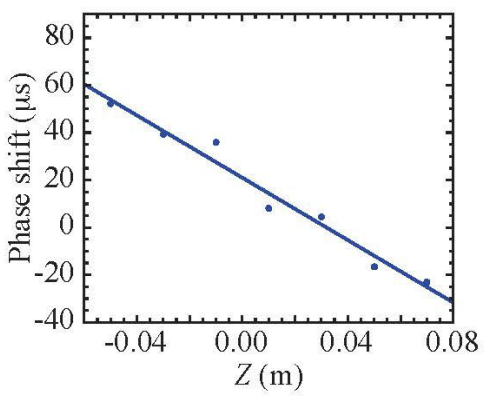

(b)

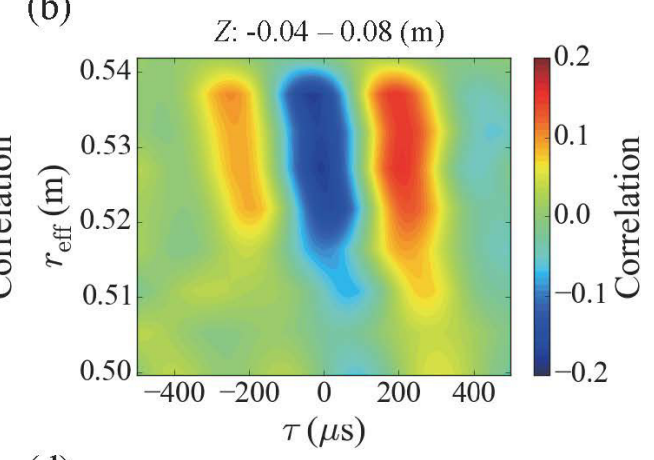

(d)

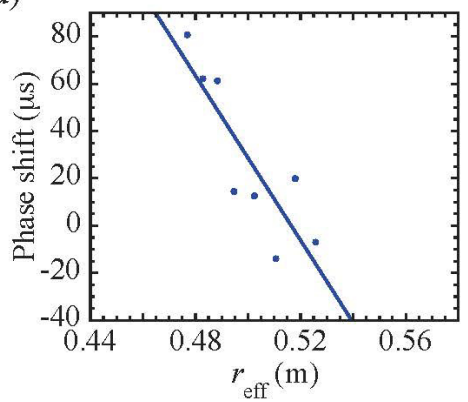

Fig. 7 Contour plot of the cross-correlation function of the low-frequency component $(1.0-3.5 \mathrm{kHz}$ ) of the density fluctuation from (a) 7 poloidal channels (group II in Fig. 2 (b)) and (b) 8 radial channels (group III) with that of the magnetic fluctuation as a reference signal. Phase shift of the cross-correlation function for (c) poloidal and (d) radial direction. 
the cross-correlation function in Fig. 7 (b) shows that the fluctuation flow the outside to the inside.

The relative phase shift among the correlation functions are plotted regarding the position of each BES channels in Figs. 7 (c) and (d) for the poloidal and radial directions, respectively. The poloidal propagation velocity $v_{\theta}$ is $\sim 1.5 \mathrm{~km} / \mathrm{s}$ with a wave number of $k_{\theta} \sim 1.7 / \mathrm{m}$. The radial propagation velocity and wave number are estimated as $v_{\mathrm{r}} \sim 0.6 \mathrm{~km} / \mathrm{s}$ and $k_{\mathrm{r}} \sim 4.3 / \mathrm{m}$, respectively.

\section{Discussion}

The spatio-temporal structure in the poloidal direction at the peak of the correlation structure of $f \sim 2.6 \mathrm{kHz}$ indicates that this mode propagates in the electron diamagnetic direction. This propagation coincides with the direction of the poloidal rotation measured with a charge exchange spectroscopy. Although $\mathrm{Z}$ direction (vertical to the equatorial plane) is almost equivalent to the poloidal direction, the relationship between the two coordinates should be estimated. On the other hand, the radial propagation direction of the mode inside the correlation peak was considered from outside to inside in the minor radius. In this work, positions in $r_{\text {eff }}$ of each sight line are evaluated by averaging the value of $r_{\text {eff }}$ weighted with the multiple value of the probe beam and the bulk plasma density on the equatorial plane. The integration effect of the sight line on the equatorial plane was estimated as $\left|r_{\text {eff }}\right| \sim 0.0 \mathrm{~m}$ at the most outside edge and as $\left|r_{\text {eff }}\right| \sim 0.1 \mathrm{~m}$ at $r_{\text {eff }} \sim 0.45 \mathrm{~m}$, and thus is gradually increasing from outside to inside.

The BES system presented in this article can set the configuration of the sight lines as well as the sampling area arbitrarily by arranging the connection between the detectors and the sight lines. Therefore, by combinig the vertically and poloidally slit-shaped sight lines to make a lattice, two-directional measurement at the same sample volume can be conducted simultaneously.

\section{Summary}

In conclusion, we have reported a new BES system which has directional sensitivity for the density fluctuation in radial or poloidal directions in LHD and the initial result of the observation. At present, this system can detect MHD activities. Using cross-correlation analysis with a supporting data of the magnetic fluctuation, the cross-correlation analysis reconstructed the radial and poloidal spatiotemporal structure regarding to MHD activity at the edge region.

\section{Acknowledgments}

The authors would like to thank the LHD team for their effort in supporting the experiment in LHD. This work is partly supported by MEXT KAKENHI Grant Number 23246164. This work is also partly supported by the National Institute for Fusion Science grant administrative budget, NIFS10ULHH021.

[1] R.J. Fonck et al., Rev. Sci. Instrum. 61, 3487 (1990).

[2] G. McKee et al., Rev. Sci. Instrum. 70, 913 (1999).

[3] D.R. Smith et al., Rev. Sci. Instrum. 81, 10D717 (2010).

[4] G.R. McKee et al., Plasma Fusion Res. 70, 913 (2007).

[5] G.R. McKee et al., Rev. Sci. Instrum. 81, 10D741 (2010).

[6] T. Oishi et al., Rev. Sci. Instrum. 75, 4118 (2004).

[7] T. Oishi et al., Rev. Sci. Instrum. 81, 10D719 (2010).

[8] S. Kobayashi et al., Rev. Sci. Instrum. 83, 10D535 (2012).

[9] T. Oishi et al., Nucl. Fusion 46, 317 (2006).

[10] A. Iiyoshi et al., Nucl. Fusion 39, 1245 (1999).

[11] D.K. Gupta et al., Rev. Sci. Instrum. 75, 3493 (2004). 